\title{
Embedding Sensitivity Analysis into PSO for Building Energy Optimization
}

\author{
Dan Hou ${ }^{1}$, Wei Yan ${ }^{2}$, Gang Liu ${ }^{1}$, Zhen Han $^{1}$ \\ ${ }^{1}$ Tianjin University, Tianjin, China \\ ${ }^{2}$ Texas A\&M University, Texas, USA
}

\begin{abstract}
Building energy optimization is highly desirable but complex in sustainable architectural design. Many studies adopt sensitivity analysis to screen the variables that have significant effects on building energy consumption, so that the solution space can be narrowed down. In that case, however, a large number of samples are used only to conduct global sensitivity analysis before optimization. In this study, an innovative building energy optimization approach is proposed, which allows the dynamic interaction between the optimization process and the sensitivity analysis. All the samples are shared by both operations to improve the optimization efficiency and effectiveness. This approach realizes a dynamic optimization process by reducing the number of variables, which enables the optimization process to evolve in conjunction with the design process. According to the result of a case study in this paper, a Particle Swarm Optimization (PSO) algorithm embedding sensitivity analysis can find the optimal solutions faster than the standard PSO.
\end{abstract}

\section{Introduction}

With the development of computing technologies, design optimization has become an important design method. The optimization technology is based on computational sciences and mathematics, as well as an understanding of the design problems. The optimization algorithms like Particle Swarm Optimization (PSO) are developed to solve the optimization problems. PSO algorithm is a metaheuristic algorithm based on the bird swarm intelligence behaviour. The PSO algorithm updates the velocities and the positions of the particles according to the information exchanged among the population. In the field of building performance optimization, sometimes the solution space can be enormous and some variables are discrete variables leading the algorithms to local optima. Many studies tried to improve the PSO algorithm to help it jump out of local optima. Angeline (1998) introduced selection operations in evolutionary algorithms to the PSO algorithm. Zhang et al. (2003) used the different mutation operators to improve the performance of the PSO algorithm. They proposed a new strategy for PSO based on orthogonal learning (OL) to help PSO discover more information that lies in two Components (Cognitive Component and Social Component) via orthogonal experimental design. This strategy can make particles to search in better directions by constructing a much promising exemplar. Niknam et al. (2010) also proposed a new mutation operator in order to improve the ability of global searching and prevent the premature convergence to the local optima. Wang et al. (2012) proposed an improved self-adaptive PSO algorithm (ISAPSO), in which the cognitive and social learning rates are changed constantly during the evolution process of the population, so the velocity direction of each particle is dynamically adjusted during the evolution process in order to keep the diversity of population and jump out of local optima. Higashi et al. (2013) introduced a Gaussian operator to mutate the velocity and position of particles in PSO. Harrison et al. (2018) introduced a speed update formula without inertia parameters to accelerate the convergence speed of the algorithm.

Sensitivity analysis (SA) methods allow the study of relationships between the uncertainty in the output of a model and different sources of uncertainty in the model input (Saltelli, 2000). Lam et al. (1996) researched the effects of different variables on building energy consumption. Heidelberg et al. (2009) made a sensitivity analysis for an office building in Denmark to measure the influence of design parameters on the total building energy demand. Shen et al. (2013) identified the most important factors with respect to building thermal and lighting energy performance so as to facilitate decision making in building design and simplify further investigation such as the optimization analysis. Yu et al. (2013) performed a sensitivity analysis on an architectural optimization problem to find the impact of 8 design variables on the building energy consumption. Gagnon et al. (2018) performed an extended sensitivity analysis of 30 design variables with respect to different performance criteria related to energy consumption and comfort, based on a TRNSYS model.

Some studies adopt sensitivity analysis to screen the variables that have the most significant effects on building energy consumption in order to narrow down the solution space. However, this processing method has two defects:

- A large number of samples are used only to conduct global sensitivity analysis. This step usually costs much time and thus offsets its improvement in optimization efficiency.

- The estimation of insensitive variables is usually fixed and absolute after the sensitivity study. When only few variables can be discarded, the sensitivity makes not much sense for improving optimization efficiency. 
In this study, an innovative building energy optimization approach is proposed, which allows the dynamic interaction between the optimization process and the sensitivity analysis. All the samples are shared by both these operations to improve the optimization efficiency and effectiveness. This approach realizes a dynamic optimization process by reducing the number of variables. The optimization process can evolve in conjunction with the design process.

\section{Principle of the PSO algorithm}

Based on the behaviour of the bird swarm, Kennedy and Eberhart (1995) proposed a parallel stochastic algorithm named particle swarm optimization (PSO) algorithm. And Shi and Eberhart (1998) introduced the inertia weight into the original PSO algorithm. This improved algorithm added the inertia weight, which stood for the degree of support for inheritance, is called the standard particle swarm optimization (SPSO). Visually, SPSO can be illustrated in Figure 1 with the meanings of the symbols explained in Table 1.

Table 1: Explanation of symbols.

\begin{tabular}{cc}
\hline Symbols & Explanation \\
\hline$i$ & Particle $i$ \\
$x_{i}(t)$ & Time $t$ \\
$p_{i}(t)$ & The location of particle $i$ at time $t$ \\
$g(t)$ & The best location particle $i$ has found \\
before time $t$ (pBest) \\
$x_{i}(t+1)$ & The best location population have found \\
before time $t$ (gBest)
\end{tabular}

So, the location and velocity of particle $i$ at time $t+1$ can be calculated as followings:

$$
\begin{gathered}
v_{i}(t+1)=w v_{i}(t)+r_{1} C_{1}\left(p_{i}(t)-x_{i}(t)\right) \\
+r_{2} C_{2}\left(g(t)-x_{i}(t)\right) \\
x_{i}(t+1)=x_{i}(t)+v_{i}(t+1)
\end{gathered}
$$

where $\mathrm{w}$ is inertia factor; $\mathrm{r} 1$ and $\mathrm{r} 2$ are random variables between 0 and $1 ; C_{1}$ is Cognitive Component and $C_{2}$ is Social Component. In SPSO, $C_{1}$ and $C_{2}$ are constants which are set at the beginning of the optimization. In this paper, $C_{I}$ and $C_{2}$ will change dynamically in the process of optimization according to the result of the sensitivity analysis. The algorithm will conduct the sensitivity analysis when the generation of the population is a multiple of five. The result of the sensitivity analysis, the rank of the variables in the order of importance, will be used to update $C_{1}$ and $C_{2}$.

Note that the criteria selection for sensitivity should be done with caution because most criteria require random and unbiased samples to guarantee the precision, whereas this is contradictory to the feature of PSO population since the individuals are generated towards the optima. Therefore, the Spearman Rank Correlation Coefficient (SRCC), which is not affected by the sample distribution, is used to evaluate the sensitivity of every variable. Sensitivity analysis determines the assembly of new individuals. In particular, the sensitive variables can be reserved as active ones which can be changed to construct the new individuals of the next generation, while the rest insensitive ones would keep their values constant in the next step. As sensitivity is varying during the optimization process, the solution space can be dynamically reduced.

In our experiments, the initial values of $C_{1}$ and $C_{2}$ are two $1 \times \mathrm{n}$ arrays whose elements are all set to be 2 , where $\mathrm{n}$ is the number of the decision variables. In every 5th generation, the sensitivity of every variable will be evaluated using SRCC using all accumulated particles, and $C_{1}$ and $C_{2}$ will update according to formula (3):

$$
C_{1}=C_{2}=\left[S_{t}^{(1)}, S_{t}^{(2)}, \cdots \cdots \cdots\right.
$$

where $S_{t}^{(k)}$ stands for the sensitivity of the $k$-th variable evaluated by all the accumulated samples obtained before time $t, t$ is the multiple of five. So, the more sensitive the variable is, the bigger scale its velocity will update, which means that the PSO algorithm will find the result faster when considering the sensitivity of the variables.

The sensitivity-based PSO algorithm established in this paper is shown as pseudo code with major steps in Table 2 , and the flow diagram of the algorithm is shown in Figure 2.

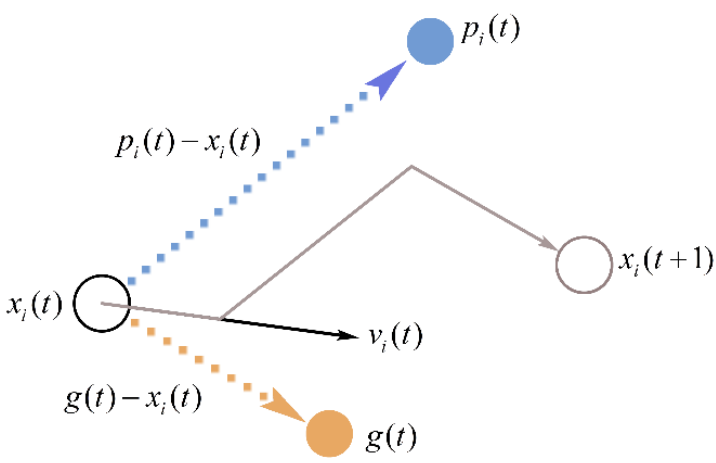

Figure 1: Schematic diagram of particle swarm optimization algorithm.

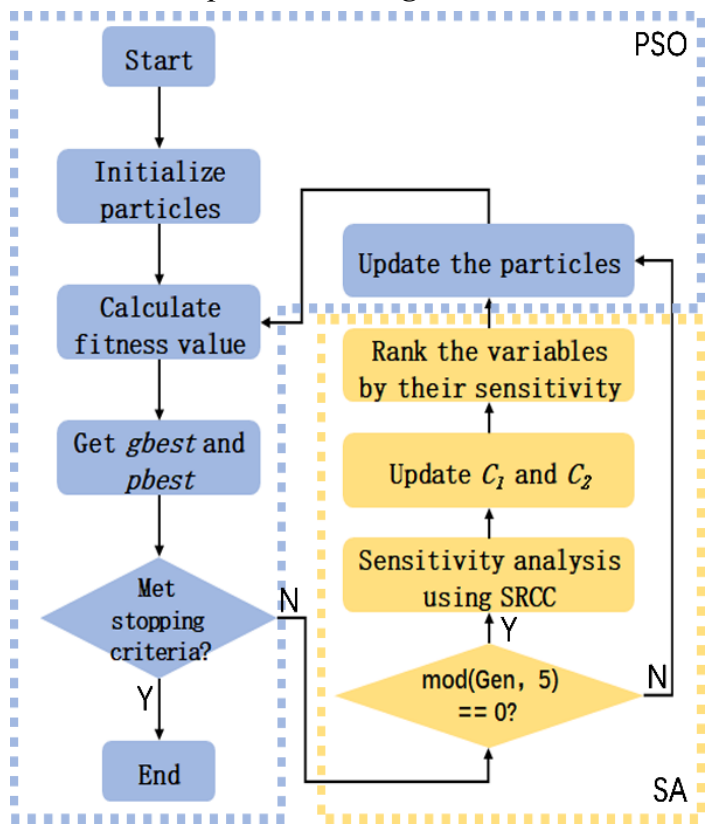

Figure 2: Flow diagram of sensitivity-based PSO. 
Table 2: Pseudo code.

\begin{tabular}{|c|c|}
\hline \multicolumn{2}{|r|}{ Algorithm 1 Sensitivity-based PSO } \\
\hline 1: & $\begin{array}{l}\text { Initialize particles (solution vectors) randomly and } \\
\text { their Velocities }=0\end{array}$ \\
\hline 2: & while not maximum iterations or minimum error \\
\hline 3: & for each particle \\
\hline 4: & Calculate fitness value \\
\hline 5: & if the fitness value $<$ pBest \\
\hline 6: & Set $p B e s t=$ current fitness value \\
\hline 7: & end \\
\hline 8: & if $\mathrm{pBest}<\mathrm{gBest}$ \\
\hline 9: & Set gBest $=$ pBest \\
\hline 10: & end \\
\hline 11: & end \\
\hline 12: & if $\bmod ($ generation, 5$)==0$ \\
\hline 13: & $\begin{array}{l}\text { Calculate and rank the sensitivity of all the } \\
\text { variables }\end{array}$ \\
\hline 14: & Update $\mathrm{C}_{1}$ and $\mathrm{C}_{2}$ \\
\hline 15: & end \\
\hline 16: & for each particle \\
\hline 17: & $\begin{array}{l}\text { Set particle Velocity = weighted sum } \\
\text { (previous velocity, difference between } \\
\text { particle's current vector and pBest's, and } \\
\text { difference between the particle's current } \\
\text { vector and gBest's) }\end{array}$ \\
\hline 18: & Update particle using Velocity \\
\hline 19: & end \\
\hline 20: & end \\
\hline
\end{tabular}

\section{Case Study}

As a case study, a specific AEC (Architecture, Engineering \& Construction) optimization task is experimented in order to prove the effectiveness of the sensitivity-based PSO algorithm.

A hypothetical office building in Tianjin is modeled using Rhino and Grasshopper. In this paper, the annual energy load per square meter of the building is chosen as the objective function to compare the performances of the SPSO and the sensitivity-based PSO. As described in Table 3, the building contains 5 rooms: Room 1 is auditorium, Room 2 is cafeteria, Room 3 is atrium, Room 4 and Room5 are workspaces. Variable1 to Variable6 are discrete variables which determine the shape of the building according to a certain design specification (each number stands for a specific ratio value of a room's width/depth, such as $1 / 2,1 / 3$, etc.). The values of the variables are set with reasonable values for architectural design. Variable 7 to Variable16 are also discrete variables which determine the location of each room. As the Figure 3 shown, the intermediary area (50 percent) of the whole site of the building will be divided into a grid to generate each room. The grid has $4 \times 4$ points intersection point. And the centers of the rooms are located at these points. For example, Room 1 shown in Figure 3 whose center is located at Point A means that Room1 Location X equals 1 and its Location Y equals 2. Variable17 to Variable29 are continuous variables which can influence the energy consumption of the building.

Table 3: Decision variables.

\begin{tabular}{|c|c|c|c|c|}
\hline Number & Decision variable & Type & Categories/Range & Description \\
\hline 1 & Building Ratio & Discrete & $0,1,2,3,4$ & the aspect ratio categories of the building \\
\hline 2 & Room1 Ratio & Discrete & $2,3,4$ & \multirow{9}{*}{$\begin{array}{l}\text { the aspect ratio categories of room } 1 \text { - } \\
\text { room5 }\end{array}$} \\
\hline 3 & Room2 Ratio & Discrete & $1,2,3,4,5$ & \\
\hline 4 & Room3 Ratio & Discrete & $1,2,3,4,5$ & \\
\hline 5 & Room4 Ratio & Discrete & $0,1,2,3,4,5,6$ & \\
\hline 6 & Room5 Ratio & Discrete & $0,1,2,3,4,5,6$ & \\
\hline 7 & Room1 Location X & Discrete & $0,1,2,3$ & \\
\hline 8 & Room2 Location $\mathrm{X}$ & Discrete & $0,1,2,3$ & \\
\hline 9 & Room3 Location X & Discrete & $0,1,2,3$ & \\
\hline 10 & Room4 Location X & Discrete & $0,1,2,3$ & \\
\hline 11 & Room5 Location X & Discrete & $0,1,2,3$ & \multirow{6}{*}{$\begin{array}{l}\text { the parameters categories which } \\
\text { determine the location of room } 1 \text { - room5 }\end{array}$} \\
\hline 12 & Room1 Location Y & Discrete & $0,1,2,3$ & \\
\hline 13 & Room2 Location Y & Discrete & $0,1,2,3$ & \\
\hline 14 & Room3 Location Y & Discrete & $0,1,2,3$ & \\
\hline 15 & Room4 Location Y & Discrete & $0,1,2,3$ & \\
\hline 16 & Room5 Location Y & Discrete & $0,1,2,3$ & \\
\hline 17 & Orientation & Continuous & {$[0, \pi / 2]$} & orientation of the building \\
\hline 18 & Window to Wall ratio $\mathrm{N}$ & Continuous & {$[0,0.25]$} & \multirow{4}{*}{$\begin{array}{l}\text { window-to-wall ratio on north, west, east } \\
\text { and south walls }\end{array}$} \\
\hline 19 & Window to Wall ratio $\mathrm{W}$ & Continuous & {$[0,0.3]$} & \\
\hline 20 & Window to Wall ratio $\mathrm{E}$ & Continuous & {$[0,0.3]$} & \\
\hline 21 & Window to Wall ratio $\mathrm{S}$ & Continuous & {$[0,0.7]$} & \\
\hline 22 & Shadow Distance & Continuous & {$[0.01,0.03]$} & distance of louver blades \\
\hline 23 & Shadow West & Continuous & {$[0,2.5]$} & width of louver blades in west \\
\hline 24 & Shadow South & Continuous & {$[0,2.222]$} & width of louver blades in south \\
\hline 25 & U Value & Continuous & {$[1,3]$} & heat transfer coefficient of glass \\
\hline 26 & SHGC & Continuous & {$[0.11,0.83]$} & solar heat gain coefficient of glass \\
\hline 27 & R Value Wall & Continuous & {$[1.5,10]$} & \multirow{3}{*}{$\begin{array}{l}\text { heat insulation coefficient of wall, roof } \\
\text { and floor }\end{array}$} \\
\hline 28 & $\mathrm{R}$ Value Roof & Continuous & {$[0.1,0.45]$} & \\
\hline 29 & $\mathrm{R}$ Value Floor & Continuous & {$[0.2,0.45]$} & \\
\hline
\end{tabular}


The bounds of those variables are set according to the related building design standards of China. For example, the bounds of the Window-to-Wall Ratio are set according to "Tianjin design standard for energy efficiency of public buildings", which requires the Window-to-Wall ratios on north, west, east and south walls of the building to be lower than $0.25,0.3,0.3$ and 0.7 , respectively.

Building Site

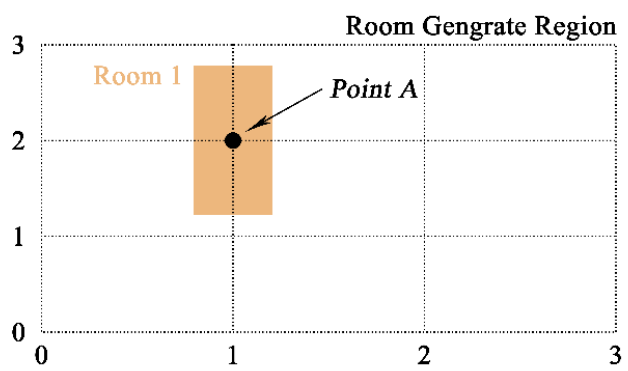

Figure 3: The diagram of the shape grammar.

In this research, the co-simulation with MATLAB \& Rhino/Grasshopper are used to solve the optimization problem. Data can be communicated between the two software tools though a CSV file. MATLAB initializes and updates the PSO velocity and position of the particles, while Rhino will generate the architectural design solutions and calculate the energy consumption with Honeybee in Grasshopper. The building model created using Rhino is shown in Figure 4.
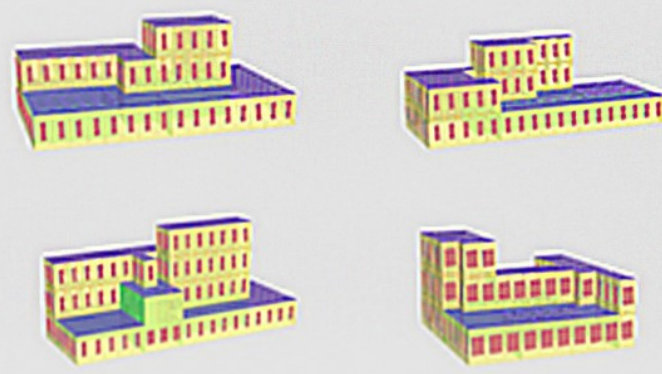

Figure 4: The building model created using Rhino.

The case study is divided into two phases. In Phase 1, the result of SPSO and five repeated experiments of the sensitivity-based PSO algorithm were compared. The parameters of each experiment are exactly the same, and the initial position of each particle in each experiment was randomized. In Phase 2, the result of SPSO and three new repeated experiments of the sensitivity-based PSO algorithm were compared. The parameters of each experiment are still the same, but the difference this time was that the initial position of each particle in each experiment was created by Latin Hypercube Sampling (LHS, instead of random sampling) which can make the sensitivity analysis more accurately (Mckay, 1979).

The parameters of PSO algorithm setting in Phase 1 and Phase 2 is shown in Table 4 (The initial values of $C_{l}$ and $C_{2}$ are two $1 \times \mathrm{n}$ arrays, $\mathrm{n}$ being the number of variables):
Table 4: Parameters setting.

\begin{tabular}{cc}
\hline Parameter & Value \\
\hline$w$ & 0.99 generation- 1 \\
$C_{1}$ & 2.0 initially, to be \\
updated \\
$C_{2}$ & 2.0 initially, to be \\
& updated \\
Mutation rate & 0.5 \\
Number of particles & 50 \\
Max generation & 50 \\
\hline
\end{tabular}

\section{Result analysis}

The results of Phase 1 are shown in Figure 5 - Figure 7. Figure 5 shows the comparison of the value of global best between SPSO and each experiment of sensitivity-based PSO; Figure 6 shows the minimum value of fitness in each generation, which can tell if the global best is caused by mutation or not. The curve will be gently if the particle evolves according to the formula (1) and formula (2) without mutation. A dramatically changed curve like Experiment 5 (the green curve) in Figure 5 must be caused by mutation. Figure 7 shows the average objective function of particles in each generation.

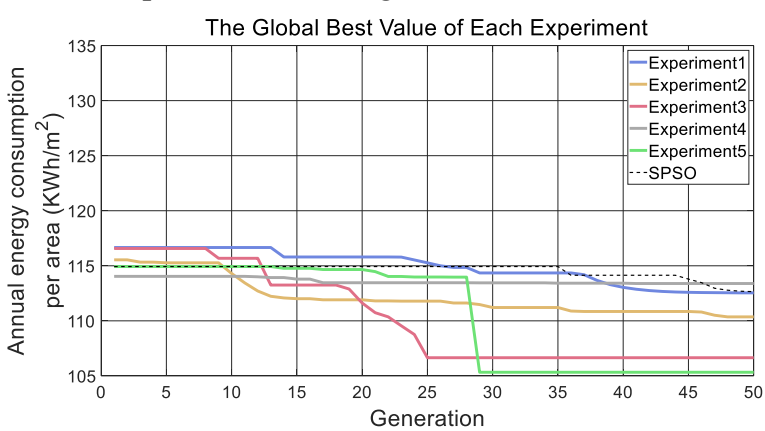

Figure 5: The comparison of global best.

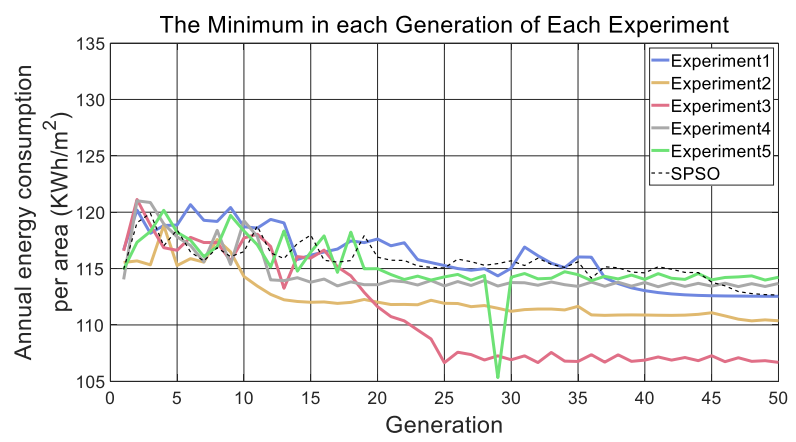

Figure 6: The comparison of minimum.

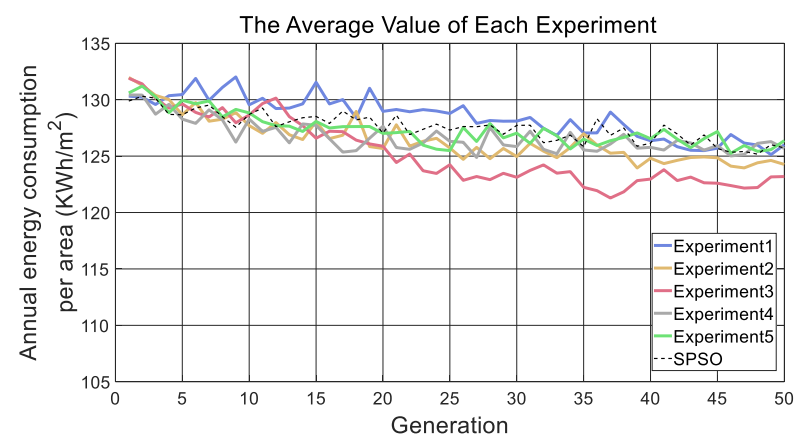

Figure 7: The comparison of average. 
Table 5: Comparison of global best in each experiment and SPSO in Phase 1.

\begin{tabular}{|c|c|c|c|c|c|}
\hline & gBest & $\begin{array}{c}\text { Generation in } \\
\text { which gBest first } \\
\text { found }\end{array}$ & $\begin{array}{l}\text { gBest caused } \\
\text { by Mutation? }\end{array}$ & Convergence? & $\begin{array}{l}\text { Method of creating } \\
\text { the initial particles }\end{array}$ \\
\hline Experiment1 & 112.54 & 45 & $\mathrm{~N}$ & $\mathrm{Y}$ & Random Sampling \\
\hline Experiment 2 & 110.36 & 47 & $\mathrm{~N}$ & $\mathrm{~N}$ & Random Sampling \\
\hline Experiment 3 & 106.65 & 25 & $\mathrm{~N}$ & $\mathrm{Y}$ & Random Sampling \\
\hline Experiment4 & 113.39 & 23 & $\mathrm{~N}$ & $\mathrm{Y}$ & Random Sampling \\
\hline Experiment5 & 105.33 & 29 & $\mathrm{Y}$ & $\mathrm{Y}$ & Random Sampling \\
\hline SPSO & 112.65 & 50 & $\mathrm{~N}$ & $\mathrm{~N}$ & Random Sampling \\
\hline
\end{tabular}

Table 5 shows the global best in each experiment and the SPSO test in Phase 1. The criterion for judging the convergence of an evolution is that the value of the global best remains unchanged for 5 generations.

According to Figure 5 - Figure 7 and Table 5, Experiment 2 and 3 achieved better results compared with SPSO. They each found a lower fitness value in an earlier generation. Experiment 5 found the global best by mutating, which was a contingent. The repeatability of the multiple group experiments could make it possible to eliminate this uncertain factor in the course of the experiment. Experiment 1 and 4 didn't perform well. The global best results they found are similar to that of the SPSO and the convergence of them also slow.

The accuracy of the sensitivity analysis is depending on the distribution of the initial particles. Uniformity and coverage are calculated to evaluate the distribution of the initial particles through Population Standard Deviation: Formula (4), (5), and (6).

$$
\Delta=\sqrt{\frac{1}{N p} \sum_{i=1}^{N p}\left(s_{i}-\bar{S}\right)^{2}}
$$

where $N p$ is the number of particles; $s_{i}$ is the minimum distance between the $i$-th particle and the other particles; $\bar{S}$ is the average of the minimum distance between each particle and the other particles. So, the lower uniformity equates to better performance.

$$
\begin{gathered}
C O V=\prod_{i=1}^{n} S D_{i} \\
S D_{i}=\sqrt{\frac{\sum_{j=1}^{N p}\left(f_{i j}-M_{i}\right)^{2}}{N p}}
\end{gathered}
$$

where $n$ is the number of the decision variables; $S D_{i}$ is the standard deviation of the $i$-th variable values of all the particles; $f_{i j}$ is the value of the $\mathrm{i}$-th variable of the $\mathrm{j}$-th particle; $M_{i}$ is the average of the $\mathrm{i}$-th variables of all the particles. So, the higher coverage equates to better performance.

This research calculated the uniformity and coverage of the particles in first ten generations of each experiment. The result is shown in Table 6.
Table 6: The uniformity and coverage of each experiment.

\begin{tabular}{lcc}
\hline & Uniformity & Coverage \\
\hline Experiment 1 & 3.1391 & $2.2360 \mathrm{e}-20$ \\
Experiment 2 & 3.0808 & $2.8362 \mathrm{e}-20$ \\
Experiment3 & 2.6473 & $3.2359 \mathrm{e}-20$ \\
Experiment4 & 3.1381 & $0.8673 \mathrm{e}-20$ \\
Experiment5 & 2.6535 & $0.7594 \mathrm{e}-20$ \\
\hline
\end{tabular}

According to the result in Table 6, Experiment 2 and 3 which performed well during the evolution have lower uniformity than Experiment 1 and 4, and higher coverage than Experiment 1, 4, and 5. So, the sensitivity of these two experiments are more accurate than Experiment 1 and 4. On the other hand, the experiment with bad coverage like experiment 1,4 and 5 can also easily be trapped into the local optima because of the wrong evolution direction.

Column 1 in Table 7 is the sequence number of the variables; Columns 2 - 6 show the ten most sensitive variables of each experiment given by the sensitivity analysis using the particles in the first ten generations.

In the project, nine training sets with different number of samples are also used in order to provide a more accurate analysis to the sensitivity of the variables. There are 40, $80,120,160,200,400,800,1000$ and 2000 samples in each of the nine training sets. The result of the sensitivity analysis will be more accurate with a bigger number of the samples. Conducting the sensitivity analysis with the nine training sets, the result of the sensitivity analysis didn't change when the number of the sample is larger than 800 . So, this research considered the result of the sensitivity analysis with 800 samples as the real result of the sensitivity analysis.

Column 7 in Table 7 shows the accurate result of the ten most sensitive variables. A gray cell in Column 2 to Column 6 means that the variable recorded in that cell also appears in Column 7. Thus, the more number of the gray cells the experiment has, the more accurate result of sensitivity analysis it obtains. According to Table 7, the ten most sensitive variables ranked by experiment 2 and 3 are closer to the accurate rank which lead these two experiments searching the solution space in a better direction.

In Phase 2, the initial positions of the particles were given by Latin hypercube sampling (LHS). LHS, which was first described by Mckay et al., is a statistical method of generating a sample of different variables. It is used to create a near-random sample from a high dimensional distribution. 
Table 7: The ten most sensitive variables.

\begin{tabular}{|c|c|c|c|c|c|c|}
\hline No & Experiment1 & Experiment2 & Experiment3 & Experiment4 & Experiment5 & Real \\
\hline 1 & $\begin{array}{c}\text { Window to Wall } \\
\text { ratio } \mathrm{N}\end{array}$ & $\begin{array}{c}\text { Window to Wall } \\
\text { ratio } \mathrm{W}\end{array}$ & Orientation & Room5 Ratio & $\begin{array}{c}\text { Window to Wall } \\
\text { ratio } \mathrm{N}\end{array}$ & SHGC \\
\hline 2 & $\begin{array}{c}\text { Window to Wall } \\
\text { ratio } \mathrm{W}\end{array}$ & Shadow Distance & $\begin{array}{l}\text { Window to Wall } \\
\text { ratio } \mathrm{W}\end{array}$ & SHGC & $\begin{array}{c}\text { Window to Wall } \\
\text { ratio } \mathrm{W}\end{array}$ & $\begin{array}{l}\text { Window to } \\
\text { Wall ratio } S\end{array}$ \\
\hline 3 & Room5 Ratio & U Value & $\begin{array}{c}\text { Window to Wall } \\
\text { ratio E }\end{array}$ & R Value Wall & Shadow West & $\mathrm{R}$ Value Wall \\
\hline 4 & Shadow Distance & $\mathrm{R}$ Value Wall & $\begin{array}{c}\text { Window to Wall } \\
\text { ratio } S\end{array}$ & R Value Roof & SHGC & $\begin{array}{l}\text { Window to } \\
\text { Wall ratio E }\end{array}$ \\
\hline 5 & SHGC & R Value Roof & R Value Roof & Room4 Ratio & Room5 Ratio & $\begin{array}{c}\text { Window to } \\
\text { Wall ratio } \mathrm{W}\end{array}$ \\
\hline 6 & R Value Roof & Orientation & R Value Floor & $\begin{array}{c}\text { Window to Wall } \\
\text { ratio } \mathrm{S}\end{array}$ & U Value & R Value Roof \\
\hline 7 & $\begin{array}{c}\text { Room2 Location } \\
\mathrm{Y}\end{array}$ & $\begin{array}{c}\text { Window to Wall } \\
\text { ratio } \mathrm{N}\end{array}$ & U Value & Room3 Ratio & Shadow Distance & U Value \\
\hline 8 & $\begin{array}{c}\text { Window to Wall } \\
\text { ratio E }\end{array}$ & Shadow West & R Value Wall & Shadow West & R Value Roof & $\begin{array}{c}\text { Room5 } \\
\text { Location X }\end{array}$ \\
\hline 9 & $\begin{array}{c}\text { Room3 Location } \\
\text { X } \\
\end{array}$ & SHGC & $\begin{array}{c}\text { Window to Wall } \\
\text { ratio } \mathrm{N}\end{array}$ & Shadow South & $\mathrm{R}$ Value Wall & $\begin{array}{c}\text { Room4 } \\
\text { Location X }\end{array}$ \\
\hline \multirow[t]{6}{*}{10} & $\begin{array}{c}\text { Room3 Location } \\
\text { Y }\end{array}$ & Shadow South & Shadow South & R Value Floor & Shadow South & $\begin{array}{l}\text { Window to } \\
\text { Wall ratio } \mathrm{N}\end{array}$ \\
\hline & & gBest & $\begin{array}{l}\text { Generation first } \\
\text { find gBest }\end{array}$ & $\begin{array}{l}\text { Mutation } \\
\text { Caused? }\end{array}$ & Convergence & $\begin{array}{c}\text { Method of } \\
\text { creating the } \\
\text { initial particle }\end{array}$ \\
\hline & xperiment1 & 109.65 & 34 & $\mathrm{~N}$ & $\mathrm{Y}$ & LHS \\
\hline & xperiment2 & 111.73 & 23 & $\mathrm{~N}$ & $\mathrm{Y}$ & LHS \\
\hline & xperiment3 & 110.22 & 31 & $\mathrm{~N}$ & $\mathrm{Y}$ & LHS \\
\hline & SPSO & 112.65 & 50 & $\mathrm{~N}$ & $\mathrm{~N}$ & Random Sampling \\
\hline
\end{tabular}

As Figure 8 shows, how many sample points to use must be determined first and whereby each sample is the only one in each axis-aligned hyperplane containing it.
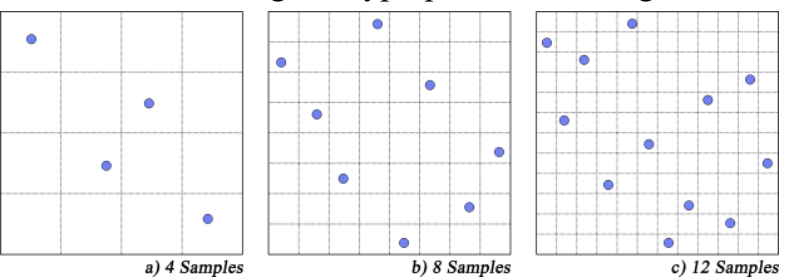

Figure 8: The principle sketch of LHS.

The results of Phase 2 are shown in Table 8 and Figure 9 - 11. Table 8 shows that the information of the global best in each experiment and SPSO. According to the result, all the three experiments achieved better results. They found lower fitness values with fewer generations.

Compared with the SPSO, the global best value of Experiment1 - Experiment 3 are 2.66\%, 0.82\% and 2.16\% lower than the SPSO. Besides, Experiment1 Experiment 3 are saving $32 \%, 54 \%$ and $38 \%$ running time. Therefore, the efficiency of the SPSO are improved significantly by embedding sensitivity analysis into it.

According the principle of the LHS, it can make sure that the initial particles have a uniform distribution and cover the solution space as much as possible. Therefore, uniformity and coverage analyses are not needed for
Phase 2. After applying LHS for initializing particles, the performance of the sensitivity-based PSO is clearly better than SPSO. In other words, the PSO algorithm has been improved when embedding the sensitivity analysis in it and initializing particles using LHS.

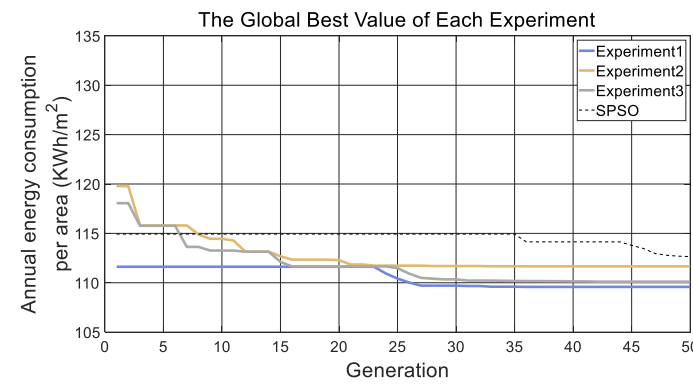

Figure 9: The comparison of global best.

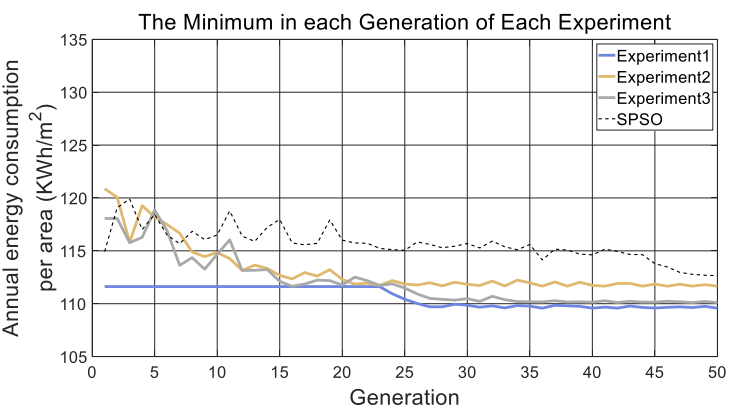

Figure 10: The comparison of minimum. 


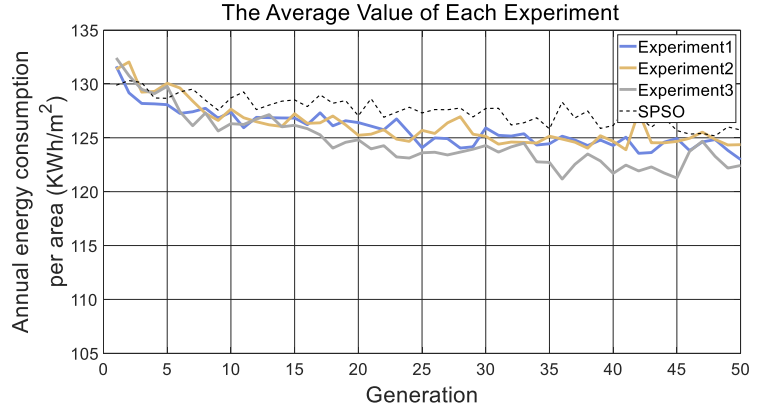

Figure 11: The comparison of average.

\section{Conclusion}

The proposed sensitivity-based approach can be an added step to embed in some optimization algorithms like PSO, Genetic Algorithm, and so on. In this study, we select a commonly used one, particle swarm optimization (PSO) algorithm, to demonstrate the effectiveness of the new approach: sensitivity-based PSO. The program is developed for this using MATLAB and Rhino.

An energy optimization problem involving various variables is created as the case study for a test of the new approach. As shown in the previous section, the new algorithm performed better than the traditional PSO algorithm. With the information exchange between sensitivity analysis and the evolution of particles, we can get the optimum solution as well as the sensitivity rank of the variables using the same samples. The integration of sensitivity analysis and optimization will greatly reduce the number of samples for sensitivity analysis prior to optimization. On the other hand, embedding sensitivity analysis in the PSO algorithm can also accelerate the evolution speed because the result of the sensitivity analysis will find the most sensitive variables and update the velocity of the particles to a direction with a larger gradient. Therefore, this approach is expected to save designers/engineers a significant amount of time in sensitivity analysis and optimization.

However, the sensitivity-based PSO algorithm still have some limitations, for example, it still has the possibility of being trapped into a local optimum. Further research is undergoing to investigate the problem. Future study also includes the study of embedding sensitivity analysis into other optimization algorithms.

\section{Acknowledgement}

This research is supported by the National Natural Science Foundation of China (Grant No. 51628803).

\section{References}

Angeline, J. (1998). Using selection to improve particle swarm optimization. IEEE International Conference on Evolutionary Computation Proceedings. Anchorage (USA), 4-9 May 1998.
Gagnon, R., Gosselin, L. and Decker, S. (2018). Sensitivity analysis of energy performance and thermal comfort throughout building design process. Energy and Buildings, 164(4), 278-294.

Harrison, R., Engelbrecht, P., and Ombuki-Berman, M. (2018). Self-adaptive particle swarm optimization: a review and analysis of convergence. Swarm Intelligence, 12(3), 187-226.

Heiselberg, P., Brohus, H., Hesselholt, A., Rasmussen, H., Seinre, E. and Thomas, S. (2009). Application of sensitivity analysis in design of sustainable buildings. Renewable Energy, 34(9), 2030-2036.

Higashi, N. \& Iba, H. (2013). Particle swarm optimization with Gaussian mutation. Proceedings of the 2003 IEEE Swarm Intelligence Symposium. Indianapolis (USA), 26 April 2003

Lam, J., and Hui, S. (1996). Sensitivity analysis of energy performance of office buildings. Building and Environment, 31(1), 27-39.

Mckay, D., Beckman, J. and Conover, W. J. (1979). Comparison of three methods for selecting values of input variables in the analysis of output from a computer code. Technometrics, 21(2), 239-245.

Niknam, T., Mojarrad, D., Meymand, Z. and Firouzi, B. B. (2011). A new honey bee mating optimization algorithm for non-smooth economic dispatch. Energy, 36(2), 896-908.

Saltelli. A., Chan K., and Scott, E.M. (2000). Sensitivity analysis. Wiley. New York (USA).

Shen, H. and Tzempelikos, A. (2013). Sensitivity analysis on daylighting and energy performance of perimeter offices with automated shading. Building and Environment, 59(1), 303-314.

Wang, Y., Zhou, J., Zhou, C., Wang, Y., Qin, H., and Lu, Y. (2012). An improved self-adaptive pso technique for short-term hydrothermal scheduling. Expert Systems with Applications, 39(3), 2288-2295.

Yu, J., Tian, L., Yang, C., Xu, X. and Wang, J. (2013). Sensitivity analysis of energy performance for highrise residential envelope in hot summer and cold winter zone of china. Energy and Buildings, 64, 264274.

Zar, J. (1972). Significance testing of the spearman rank correlation coefficient. Publications of the American Statistical Association, 67(339), 3.

Zhang, J. \& Xie, F. (2003). DEPSO: Hybrid particle swarm with differential evolution operator. 2003 IEEE International Conference on Systems, Man and Cybernetics. Washington (USA), 8 October 2003 